\title{
Approche méthodologique pour distinguer un ensemble lésionnel fiable de la pratique cavalière. Exemple du squelette de la tombe 11 du site de « La Tuilerie » à Saint-Dizier (Haute-Marne), vi è siècle
}

\author{
Methodological considerations in the identification of specific osteological markers of horse \\ riding. The example of the skeleton from burial 11, "La Tuilerie", Saint-Dizier (Haute-Marne), \\ 6th century AD
}

\author{
C. Baillif-Ducros - M.-C. Truc $\cdot$ C. Paresys $\cdot$ S. Villotte \\ Reçu le 4 octobre 2010 ; accepté le 14 septembre 2011 \\ (C) Société d'anthropologie de Paris et Springer-Verlag France 2011
}

\begin{abstract}
Résumé En archéologie, la pratique cavalière est abordée à partir de sources iconographiques ou écrites, de vestiges matériels (mors, éperons et étriers), d'ossements de chevaux et, parfois, de vestiges humains. En anthropologie biologique, plusieurs marqueurs osseux de cette pratique (parfois regroupés sous l'intitulé « syndrome du cavalier ») ont été reconnus. Toutefois, l'analyse des marqueurs osseux d'activité (MOA) nécessite le respect de règles méthodologiques strictes qui n'ont pas toujours été suivies dans les études antérieures de la pratique cavalière. L'étude du squelette du jeune sujet [20-39 ans] du site de « La Tuilerie » à Saint-
\end{abstract}

C. Baillif-Ducros $(\bowtie)$

Institut national de recherches archéologiques préventives (Inrap),

32, rue Delizy, F-93694 Pantin cedex, France

e-mail : cbaillifducros@yahoo.com

C. Baillif-Ducros $\cdot$ S. Villotte $(\bowtie)$

UMR 5199-PACEA, laboratoire d'anthropologie des populations du passé, université Bordeaux-I, bâtiment B8, avenue des

Facultés, F-33405 Talence, France

e-mail : s.villotte@bradford.ac.uk

M.-C. Truc $(\bowtie) \cdot$ C. Paresys $(\bowtie)$

Institut national de recherches archéologiques préventives (Inrap),

38, rue des Dats-ZI, F-51520 Saint-Martin-sur-le-Pré, France

e-mail : marie-cecile.truc@inrap.fr, cecile.paresys@inrap.fr

M.-C. Truc

Centre Michel-de-Boüard, Centre de Recherches Archéologiques et Historiques Anciennes et Médiévales (CRAHAM - UMR 6273, GTR Inhumations en contexte religieux), université de

Caen Esplanade-de-la-Paix, F-14000 Caen, France

S. Villotte

Biological Anthropology Research Centre,

Division Archaeological, Geographical and Environmental Sciences, Phoenix SW building, University of Bradford, Bradford,

West Yorkshire, BD7 1DP, United Kingdom
Dizier (Haute-Marne), probablement un guerrier de haut rang de l'époque mérovingienne, ouvre de nouvelles pistes de recherche pour distinguer un ensemble fiable des modifications associées à cette pratique. Ce jeune individu présente des lésions et une adaptation osseuse structurelle, l'ovalisation de l'acetabulum, probablement liées à la pratique équestre. Cette étude confirme l'intérêt de mettre en place un protocole d'étude stricte associant l'utilisation conjointe des données archéologiques, anthropologiques et médicales pour distinguer des critères spécifiques de reconnaissance de la pratique cavalière. Pour citer cette revue : Bull. Mém. Soc. Anthropol. Paris 24 (2012).

Mots clés Syndrome du cavalier · Acetabulum · Marqueurs osseux d'activité · Âge au décès · Saint-Dizier · Époque franque

Abstract In archaeology, horseback riding in past societies is investigated through iconographic and textual sources, artefacts (bits, spurs and stirrups), horse skeletons and sometimes human remains. Biological anthropologists have identified a number of bone changes associated with riding (sometimes collectively referred to as in "horse riding syndrome"), but these studies have not always taken the limitations and pitfalls of osteological markers into account in their analyses. The study of the young adult from the "La Tuilerie" site at Saint-Dizier (Haute-Marne), probably a high ranking warrior from the Frankish period, has opened up new avenues for studies aiming to identify a set of bone changes that can reliably be associated with horseback riding. This individual presents pathological conditions and structural adaptations (elongated acetabula) that are probably related to riding. Our study confirms the need to establish a strict study protocol for the combined use of archaeological, anthropological and medical data to distinguish specific 
osteological markers of horseback riding. To cite this journal: Bull. Mém. Soc. Anthropol. Paris 24 (2012).

Keywords Horse riding syndrome · Acetabulum · Occupational stress markers · Age-at-death · Saint-Dizier · Frankish period

\section{Introduction}

Le cheval, par sa domestication et son dressage, a permis à l'homme de multiplier par 10 sa vitesse de déplacement et de s'arroger une nouvelle maitrise du temps et de l'espace [1]. Cette noble conquête de l'homme a laissé une empreinte indélébile dans l'histoire. La domestication du cheval, qui se traduit en archéologie par la présence de traces d'usure typiques sur les prémolaires [1,2], pourrait également avoir laissé des traces spécifiques sur le squelette des cavaliers. C'est en tout cas ce qu'affirment certains auteurs qui ont reconnu des marqueurs osseux de la pratique équestre $[3,4]$. L'étude des marqueurs osseux d'activité (MOA) présente de nombreux écueils et limites [5-7], et les travaux anthropologiques concernant la pratique cavalière ne font pas exception. Dans ces études, la place du cheval au sein des sociétés, les évolutions technologiques de la cavalerie ainsi que les données médicales sur les cavaliers actuels sont bien souvent négligées. L'objectif de cet article est d'illustrer l'importance de l'association des données archéologiques, médicales et anthropologiques afin de permettre une meilleure reconnaissance de la pratique cavalière des populations du passé.

\section{Pratique cavalière des populations du passé}

Les plus anciennes traces archéologiques de la domestication du cheval ont été découvertes sur le site énéolithique de Botaï (steppe nord du Kazakhstan) datées de 3500 avant Jésus-Christ (av J.-C.) [1].

Dès l'époque protohistorique, le cheval est l'animal emblématique des élites sociales de l'Europe de l'Ouest. Cette monopolisation du cheval par "les privilégiés de l'Ouest » perdure pendant les siècles suivants [2]. Pour les civilisations de l'Est, notamment pour les peuples cavaliers (Scythes, Huns, Magyars ou Mongols), le cheval s'accorde à un mode de vie tel que le nomadisme ou la pastorale [8].

La présence d'un squelette de cheval associé à un ensemble funéraire renvoie l'hypothèse que ce dernier ait un lien avec les sujets inhumés. Toutefois, la seule présence d'ossements de chevaux ne suffit pas à démontrer la pratique équestre. En revanche, le dépôt d'objet lié à l'équipement du cheval au sein des sépultures est un élément important pour établir un lien entre le défunt et la pratique cavalière.
Les tombes mises au jour dans le département des Ardennes (la tombe $\mathrm{n}^{\mathrm{o}} 68$ de Charleville-Mézières/Manchester, la tombe $\mathrm{n}^{\mathrm{o}} 12$ de Thugny-Trugny/Côte de la Tombe) et de Saint-Denis (tombe 16.2371) en sont des exemples parfaits [9-12]. Chacune d'elles comporte un mors ou un éperon. De plus, ces tombes appartiennent toutes à la période mérovingienne. Cette dernière est une époque propice pour la recherche sur la pratique cavalière en raison de la richesse et du type de mobilier archéologique qui peut être associé au défunt.

L'équipement du cheval de monte évolue et se complète au cours du temps. Le mors est le premier élément qui constitue cette panoplie, alors que l'étrier en est le dernier. L'évolution, les transformations, en particulier celles de la selle, et l'ajout de nouveaux éléments d'harnachement, l'étrier en particulier (début $\mathrm{VII}^{\mathrm{e}}$ siècle en Occident) [13], démontrent une recherche pour la bonne assise du cavalier, notamment au combat [14]. Toutefois, ce confort n'est pas accepté dès l'antiquité. En effet, l'auteur grec Xénophon $\left(\mathrm{v}^{\mathrm{e}}\right.$ siècle av J.-C.) refuse que le cavalier se tienne sur l'ephippium ${ }^{1}$ comme sur un siège : " le soldat ne doit pas abuser de l'ephippium pour prendre ses aises et se relâcher de la position correcte qui est de rigueur dans une troupe bien dressée. La bonne assiette n'est pas de se tenir comme sur un siège mais plutôt comme si on était debout les jambes écartées [15] ». Cette citation, ce besoin d'innover et l'introduction de nouveaux éléments contribuant au confort du cavalier dans son exercice permettent d'entrevoir les traumatismes causés par la monte. Et, plus généralement, d'appréhender l'influence du matériel équestre et de l'assise sur le corps du cavalier. Par conséquent, la reconnaissance de marques spécifiques sur ce dernier, causées par cette pratique, pourrait être un élément important pour la recherche des premières traces de la domestication du cheval.

\section{MOA liés à la pratique équestre ou " syndrome du cavalier » : travaux antérieurs et limites}

L'étude du « syndrome du cavalier » est principalement représentée par les travaux de Pálfi sur les cavaliers archers hongrois et de Miller sur les tribus Omaha et Ponca (Amérique du Nord) $[3,4,16]$. Dans la lignée de ces travaux princeps se sont succédé les études d'Alduc-le-Bagousse et al., de Blondiaux, de Courtaud et Rajev, de Erickson et al., de Charlier, de Fornaciari et al. et de Üstündag et Deveci [1723]. Ces études antérieures trouvent leur validité premièrement dans la répétition, pour plusieurs individus, du même « pattern » lésionnel et deuxièmement dans le contexte

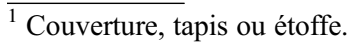


archéologique. Les principales marques reconnues correspondent à des modifications de l'aspect osseux des enthèses (enthésopathies ou élargissements des zones d'insertion), des atteintes articulaires et de l'ovalisation de l'acetabulum (Tableaux 1 et 2). On remarque que ces travaux privilégient les insertions musculaires sur la ligne âpre et plus généralement sur le fémur. Les muscles adducteurs et les muscles fessiers sont particulièrement bien représentés.

Ces études de «MOA » présentent toutes un intérêt évident pour la reconnaissance de la pratique cavalière en anthropologie, mais ne sont malheureusement pas exemptes de limites.

L'âge est un paramètre majeur dans l'apparition des modifications osseuses au niveau des articulations et des enthèses. Il semble impossible de distinguer, chez un individu âgé les atteintes microtraumatiques éventuellement apparues durant sa jeunesse, des modifications associées à la sénescence [5-7]. Les auteurs n'ont jamais réellement considéré ce point fondamental, et il est intéressant de noter que la plupart des cavaliers reconnus par les anthropologues sont des sujets âgés. Ce biais est parfaitement illustré par l'étude de Fornaciari et al. [22] : plus l'individu est âgé, plus la probabilité qu'il s'agisse d'un cavalier augmente. Par ailleurs, les désordres métaboliques ou inflammatoires ainsi que les anomalies ostéoarticulaires perturbant la biomécanique normale ne sont pas pris en compte dans les études $[5,24]$. Pálfi et Dutour sont les seuls à mentionner l'exclusion des cas de maladie hyperostosique, source d'enthésopathies non mécaniques $[3,25]$.

L'ensemble des études anthropologiques s'accorde pour considérer les muscles adducteurs comme les muscles du cavalier (Tableau 1) : leurs insertions marquées sur la ligne âpre indiqueraient une sollicitation importante. Il est fort probable que ces enthèses soient des insertions fibreuses, et

Tableau 1 Les enthésopathies liées au « syndrome du cavalier » / Enthesopathies related to "horse riding syndrome"

\begin{tabular}{|c|c|c|}
\hline Zones d'insertion & Muscles & Auteurs \\
\hline Tubérosité ischiatique & $\begin{array}{l}\text { mm. biceps fémoral, semi-tendineux, } \\
\text { semi-membraneux, grand adducteur }\end{array}$ & [3] \\
\hline Ligne âpre & $\mathrm{mm}$. vaste latéral ${ }^{\mathrm{a}}$, grand, long et court adducteur & {$[3]^{\mathrm{a}},[18,19]$} \\
\hline Tubercules supracondylaire latéral et médial & mm. gastrocnémiens (chef latéral et médial ${ }^{\mathrm{a}}$ ) & {$[3],[4]^{\mathrm{a}}$} \\
\hline Petit trochanter & m. psoas iliaque & {$[3]$} \\
\hline Ligne pectinée du fémur & m. pectiné & [3] \\
\hline Épine iliaque postérosupérieure & m. grand fessier & [3] \\
\hline Tubérosité glutéale & mm. grand adducteur ${ }^{\mathrm{a}}$, grand fessier ${ }^{\mathrm{b}}$ & {$[3]^{\mathrm{a}},[18]^{\mathrm{b}}$} \\
\hline Fosse iliaque externe & mm. moyen et petit fessier & [3] \\
\hline Grand trochanter & mm. moyen et petit fessier & {$[3,18]$} \\
\hline Tubercule de l'adducteur & m. grand adducteur & [3] \\
\hline Patella & m. quadriceps fémoral & [21] \\
\hline Fosse trochantérique & m. obturateur externe & {$[18]$} \\
\hline Tubérosité calcanéenne & m. triceps sural & {$[21]$} \\
\hline
\end{tabular}

Tableau 2 Les modifications articulaires liées au "syndrome du cavalier » / Joint alterations related to "horse riding syndrome"

\begin{tabular}{|lll|}
\hline Zones anatomiques & Modifications articulaires & Auteurs \\
\hline Acetabulum & Extension, élongation et ovalisation & {$[3,4,19,20,23]$} \\
Tête fémorale & Empreinte iliaque & {$[3,4]$} \\
Rachis, acetabulum, MTT I ${ }^{\mathrm{a}}$, fovéa ${ }^{\mathrm{b}}$ & Arthrose & {$[4]^{\mathrm{a}},[18]^{\mathrm{b}},[21]$} \\
Rachis thoracique & Dystrophie rachidienne de croissance ou maladie & {$[23]$} \\
& de Scheuermann & {$[18]$} \\
Rachis lombaire & Hyperlordose lombaire & \\
\hline${ }^{\mathrm{a}}$ L'auteur mentionne uniquement cette pièce osseuse & / The author mentions only this bone or area. \\
b Idem / Ditto. & & \\
\hline
\end{tabular}


les remodelages concernant ces insertions diaphysaires sont, à l'heure actuelle, malheureusement trop peu documentés pour être interprétés correctement comme des MOA $[6,7,26]$. Plus important encore, les lésions des muscles adducteurs décrites en médecine sportive concernent l'insertion sur la branche ischiopubienne et le pubis et non l'insertion sur le fémur [27,28].

Un autre élément ostéologique est également souvent étudié : l'acetabulum. Son ovalisation est attestée pour chacun des sujets désignés comme de probables cavaliers $[3,4,19,20,23]$. La relation entre l'ovalisation de l'acetabulum et la pratique cavalière a été étudiée en détail par Erickson et al. [20]. Ces auteurs ont montré que l'acetabulum présente au niveau de son bord antérosupérieur un étirement vers le haut chez la population en contact avec le cheval.

\section{Jeune sujet de la sépulture 11 du site de « La Tuilerie » à Saint-Dizier (Haute-Marne), $\mathrm{VI}^{\mathrm{e}}$ siècle}

\section{Présentation du site}

Le lieu-dit « La Tuilerie » est localisé au sud de l'agglomération Bragarde, dans un secteur riche en données archéologiques [29]. En effet, cet endroit est occupé de façon continue de la protohistoire au début du Moyen Âge : $\mathrm{du}_{\mathrm{I}}^{\mathrm{er}}$ au $\mathrm{v}^{\mathrm{e}}$ siècle s'implante une villa dont la pars urbana a été partiellement fouillée [30,31]. Sur ses ruines se développent une nécropole mérovingienne et un vaste et dense habitat médiéval abandonné vers le $\mathrm{XII}^{\mathrm{e}}$ siècle [31-33].

Les tombes se trouvent précisément en limite sud de cet habitat, dans une zone peu dense en vestiges, comme si les successeurs avaient respecté ce lieu, peut-être signalé par un tertre. Les défunts, deux hommes et une jeune femme, ont été inhumés avec un riche mobilier qui permet de dater l'ensemble entre le deuxième quart et la fin de la première moitié $\mathrm{du} \mathrm{VI}$ e siècle (Fig. 1). Chaque sépulture masculine consiste en une chambre coffrée de type Morken : dans la partie nord, le défunt repose dans un cercueil avec vêtements, accessoires, épée et scramasaxe [34,35]. La hache et le bouclier ont été placés contre le cercueil. Le reste de la chambre est réservé à la vaisselle de bronze et de verre. Les armes les plus volumineuses, telles que lance et angon, ont été placées sur le couvercle de la chambre. Cette panoplie d'armes très complète signe à la fois le statut de guerrier et le haut rang social des deux hommes. En effet, si dans les tombes mérovingiennes " classiques ", la découverte de haches, de scramasaxes et de boucliers est courante, l'ajout d'une épée, d'une lance et d'un angon est en revanche révélateur d'une élite. La jeune femme reposait dans un cercueil placé dans une fosse plus petite. Ses nombreux bijoux : fibu- les en argent et grenats, perles en matériaux variés, bague en or, etc., attestent là encore de son rang social très élevé.

Une sépulture de cheval (Fig. 2) a été découverte 5,50 m à l'est des tombes humaines. Ce mâle adulte, âgé d'environ huit ans, est d'une grande taille pour l'époque. Ses prémolaires présentent des traces attribuables au port d'un mors, indiquant qu'il devait s'agir d'un cheval de monte [36]. Aucune trace de pathologie, ni de dépeçage n'a été relevée sur ses ossements. Il faut sans doute voir dans cette inhumation soignée la volonté de réunir le propriétaire et sa monture. La sépulture était vide de mobilier, mais un mors retrouvé dans la sépulture du jeune homme (sépulture 11) pourrait indiquer que le cheval lui appartenait. L'inhumation de chevaux - entiers ou découpés - au sein des nécropoles, coutume germanique déjà rapportée par Tacite au $\mathrm{I}^{\mathrm{er}}$ siècle, se développe surtout dans la seconde moitié $\mathrm{du}_{\mathrm{VI}}^{\mathrm{e}}$ et au $\mathrm{VII}^{\mathrm{e}}$ siècle [37,38]. Fréquente sur la rive droite du Rhin, cette pratique reste marginale en Gaule mérovingienne chrétienne, où elle est surtout localisée entre Somme et Escaut, c'està-dire au cœur des premiers royaumes francs [39]. Ce type d'inhumation aurait un rôle psychopompe, le cheval aidant le défunt à se rendre dans l'au-delà [40]. Le sacrifice d'un animal a priori en bonne santé reflète le prestige et le haut rang social du défunt, au même titre que le dépôt de vaisselle de luxe dans sa tombe.

Ces tombes affichent des attributs germaniques qui tranchent sur les rites funéraires de la région : chambres funéraires de type Morken, inhumation d'un cheval, présence d'objets de type franc, etc. Ces particularités permettent d'attribuer la découverte au faciès archéologique des tombes dites « de chefs francs » du début du vi ${ }^{\mathrm{e}}$ siècle. Ce faciès est caractérisé en Europe du Nord par une cinquantaine de riches tombes masculines qui présentent entre elles de fortes similitudes dans les rites funéraires et l'agencement des dépôts, traduisant la volonté de montrer l'appartenance du défunt à une élite sociale particulière [41]. Les tombes masculines comportent généralement une épée au pommeau prolongé par un double anneau, symbole de liens guerriers d'homme à homme noués au sein de cette élite $[42,43]$. Tel est le cas pour l'épée du jeune homme (sépulture 11). La répartition des tombes reflète la conquête franque : les tombes les plus précoces occupent le centre du royaume franc alors que les suivantes - dont celles de Saint-Dizier - sont situées sur les marges. Leur rôle militaire et stratégique semble fort probable : pour consolider sa conquête, Clovis puis ses descendants durent fixer une élite guerrière, s'assurant ainsi le contrôle sur des terres nouvellement conquises [41]. La présence d'une élite guerrière à Saint-Dizier, qui à l'époque est un habitat sans importance notable, peut avoir un lien avec sa situation stratégique, proche de la frontière avec la Bourgogne (jusqu'en 534). Elle peut aussi résulter d'une volonté d'avoir la mainmise sur le minerai de fer, présent en abondance dans les environs et largement exploité aux siècles suivants [44]. 


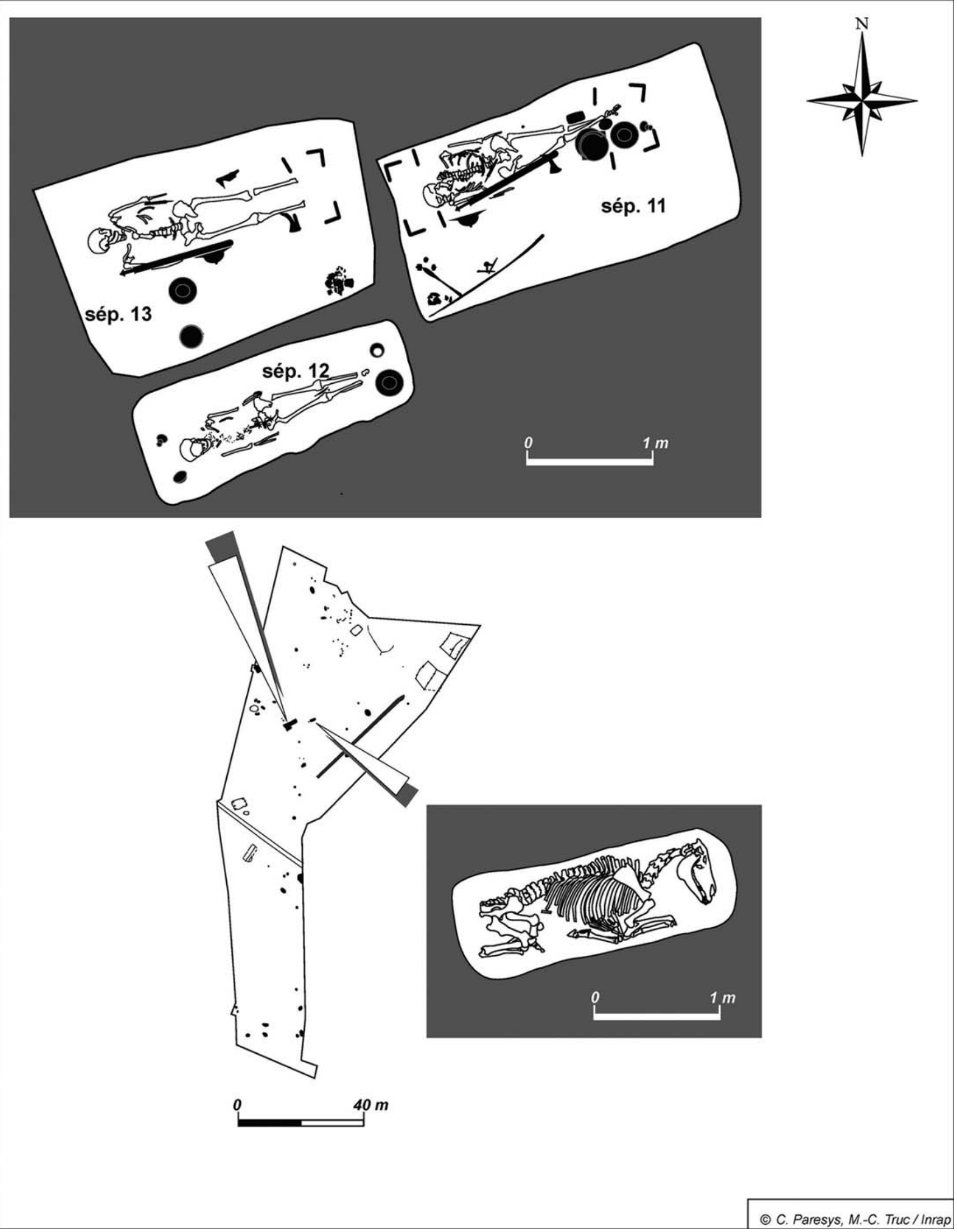

Fig. 1 Plan du gisement funéraire / Plan of the burial site 


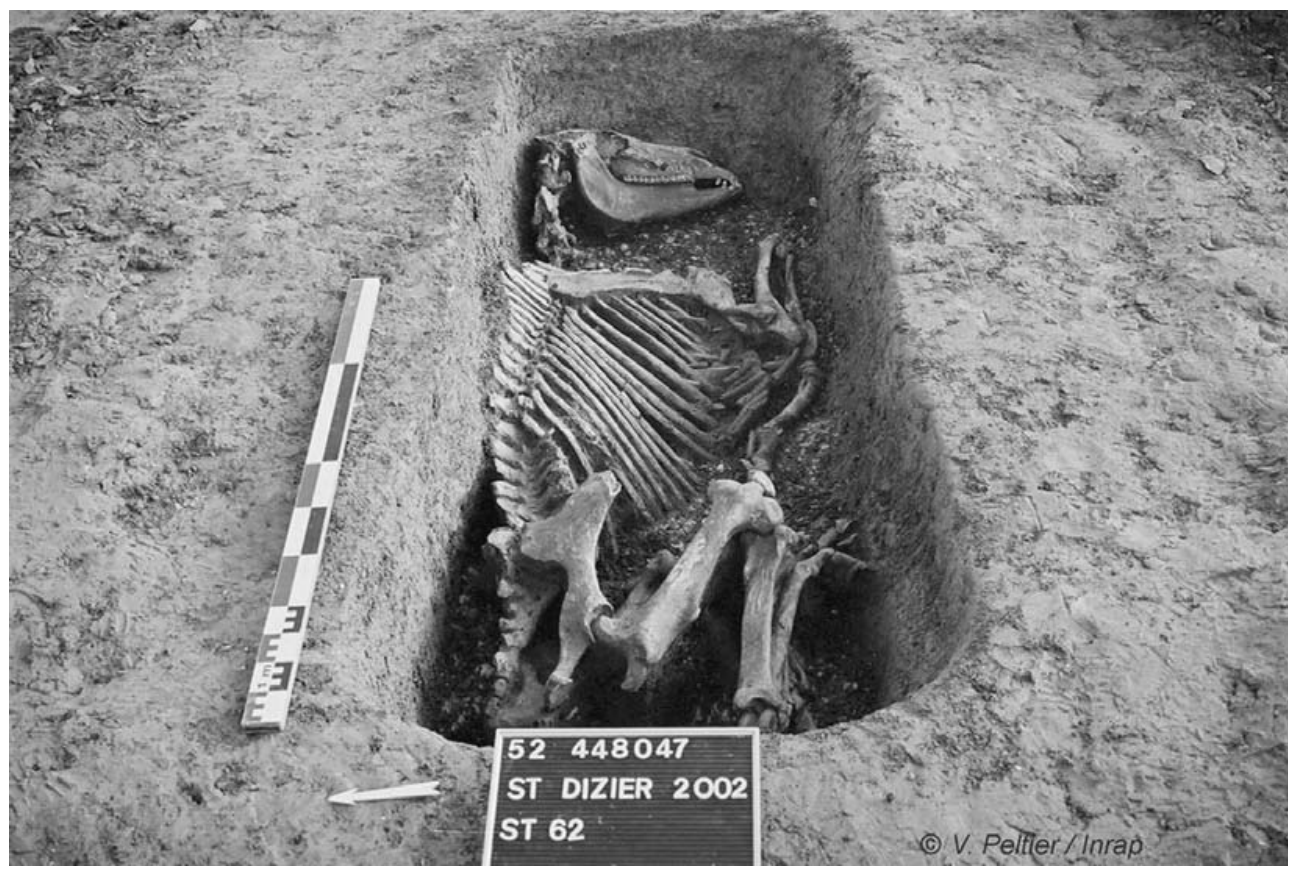

Fig. 2 Tombe de cheval / Horse burial

\section{Matériel}

Les trois inhumés de Saint-Dizier sont deux hommes et une femme $[45,46]$. L'âge du premier sujet masculin est compris entre 20 et 39 ans (sépulture 11) [47,48]. L'autre homme est plus âgé, autour d'une cinquantaine d'années (sépulture 13). Une adolescente de 17-19 ans (sépulture 12) était inhumée à leurs côtés [49,50].

Les statures estimées pour les deux hommes sont assez proches : $182 \mathrm{~cm}$ pour celui de la sépulture 11 et $178 \mathrm{~cm}$ pour celui de la sépulture 13 [51]. Celle de l'adolescente avoisine les $165 \mathrm{~cm}$, mais les épiphyses des os longs n'étant pas fusionnées aux diaphyses, sa croissance n'était probablement pas tout à fait achevée [51]. Ces statures sont supérieures d'une dizaine de centimètres à celles d'une population villageoise contemporaine (Savigny-sur-Ardres) [52].

Les ossements de ces trois défunts renvoient une image d'individus relativement préservés, avec une faible présence des indicateurs non spécifiques de stress et des maladies dégénératives (arthrose et enthésopathies), hormis les hypoplasies linéaires de l'émail dentaire, présentes avec une intensité légère à moyenne sur les trois sujets, quoique plus marquées et plus nombreuses chez la jeune fille [29]. Le squelette de l'homme de la sépulture 13 présente plusieurs articulations anormalement soudées. L'association de ces éléments - fusion sacro-iliaque droite, bloc vertébral de deux vertèbres thoraciques, ossification d'un ligament costosternaire à gauche - est généralement interprétée comme l'indice d'une maladie hyperostosique [29,53]. La recherche d'une éventuelle parenté biologique entre ces trois sujets n'est pas confirmée par l'analyse des caractères discrets, dont un seul est partagé par deux défunts [29,54]. En revanche, les analyses $\mathrm{ADN}^{2}$ ont mis en évidence l'appartenance possible à une même lignée maternelle pour les deux jeunes sujets (sépultures 11 et 12). Toutefois, d'autres formes de liens (sociaux par exemple) ne sont pas exclues.

L'examen anthropologique et pathologique de ces individus témoigne donc d'un état sanitaire globalement bon, faisant référence à une population plutôt favorisée, épargnée par les agressions environnementales fréquentes dans d'autres populations villageoises contemporaines. L'observation d'une pathologie pouvant être liée à une alimentation riche et abondante va également dans ce sens.

L'étude s'est orientée vers le sujet de la sépulture 11 pour plusieurs raisons :

- le défunt appartient à l'élite guerrière franque. La panoplie d'armes très complète confirme à la fois le statut de guerrier et le haut rang social du sujet ;

- son jeune âge. La classe d'âge [20-39] permet d'étudier les anomalies de croissance liées à une activité intense dès l'enfance, les atteintes microtraumatiques (arthrose et enthésopathies) apparues durant sa jeunesse et elle exclut d'elle-même les faux-positifs liés à la sénescence ;

- le dépôt funéraire associé au sujet comprend un mors à branches de cheval : cet élément d'harnachement évoque indiscutablement la pratique équestre.

\footnotetext{
2 Résultats obtenus par le Pr J.J. Cassiman (Center for Human Genetics/Forensic Medicine, Louvain, Belgique).
} 


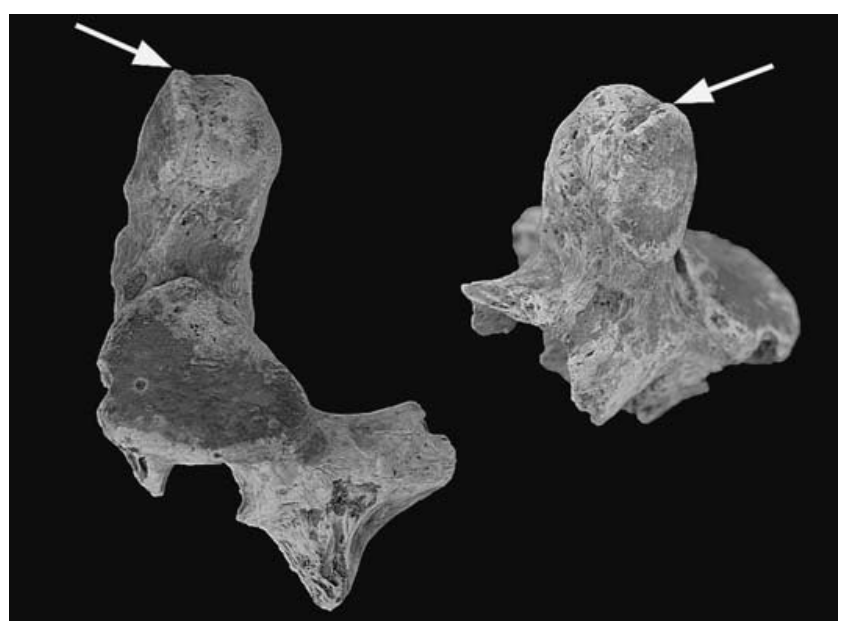

Fig. 3 Déformation mineure du contour de la dent de l'axis / Minor deformation of the dens axis

Les squelettes de la jeune adolescente (sépulture 12) et du sujet âgé (sépulture 13) ont été exclus en raison d'une mauvaise conservation osseuse pour la première et du diagnostic d'une possible maladie hyperostosique (source de fauxpositifs) pour le second.

\section{Résultats}

Le squelette du jeune homme (sépulture 11) est en très mauvais état de conservation. De nombreuses pièces anatomiques sont absentes ou fragmentées. Les branches ischiopubiennes et les pubis sont manquants. On observe une fracturation, pour chacun des os coxaux, au niveau de la jonction de la branche supérieure du pubis et de l'acetabulum. Le rachis et la partie proximale des fémurs sont, également, très mal conservés. Les vertèbres cervicales et thoraciques présentent une déformation mineure au niveau des contours de l'articulation atlanto-odontoïdienne (Figs. 3,4) et des articulations postérieures (légères ostéophytoses marginales) (Fig. 5). Sur les corps de deux vertèbres cervicales (rang indéterminé), on note la présence, au centre, de traces d'une ostéochondrose intervertébrale modérée (Fig. 6). De plus, l'étude des vertèbres thoraciques (six présentes et de rang indéterminé) et lombaires (L4 uniquement) ne révèle pas la présence de lésion typique en rapport avec une dystrophie rachidienne de croissance (DRC) ou d'une hyperlordose. Par ailleurs, chacun des acetabula du sujet présente une élongation du bord antérosupérieur (Fig. 7). Les diamètres vertical (VEAC-M22 [55]) et horizontal (HOAC-M22 [55]) maximaux des acetabula ont été mesurés. Les points de mesure sont situés sur le bord de l'acetabulum (ni internes ni externes). La hauteur $\left(\operatorname{VEAC}^{3}\right)$ est mesurée dans un axe prolongeant l'axe général de l'ischium, et la largeur maximale (HOAC) est, quant à elle, mesurée perpendiculairement à ce dernier. Ces mesures établies et estimées sur les acetabula (Tableau 3) indiquent un rapport hauteur/largeur important : 1,11 pour le coxal droit et de 1,07 pour le coxal gauche. Comparés à une collection ostéologique de référence [56], ces rapports apparaissent bien au-dessus de la médiane $^{4}$ (Fig. 8). Les têtes fémorales présentent un creusement et un élargissement marqués des fovéas avec une légère ostéophytose périfovéale en bourrelet (Fig. 9). Les mesures des longueurs et largeurs de chacune des fovéas (Tableau 4) ont été prises à la marge externe de ce bourrelet.

\section{Discussion}

D'un point de vue archéologique, l'ensemble des critères pour attester de la pratique cavalière est réuni : l'individu appartient à l'élite mérovingienne pour laquelle l'usage du cheval est connu, le mobilier associé au défunt est un mors de cheval et les traces d'usure retrouvées sur les prémolaires de l'animal le désignent comme un cheval de monte. Sur le plan anthropologique, l'âge du sujet est un critère décisif : il permet de considérer les modifications des contours articulaires observées sur le rachis, l'ostéochondrose intervertébrale sur le corps des deux vertèbres cervicales et les ostéophytoses périfovéales comme étant probablement la conséquence de microtraumatismes. De plus, ces atteintes peuvent être discutées à la lumière des données médicales sur les pathologies des cavaliers actuels. La localisation, atypique, de l'ostéochondrose intervertébrale sur le rachis cervical peut ainsi s'expliquer par une hypermobilité d'un segment du rachis cervical par rapport à un autre [57]. Les modifications des contours articulaires observées sur le rachis peuvent être la conséquence de chocs répétés, de chutes ou d'un mauvais encaissement de saut [57]. Les ostéophytoses périfovéales sont également observées chez les cavaliers actuels [27,58]. Toutefois, Auvinet ne considère pas cette lésion comme spécifique du cavalier mais plus largement comme celle des sportifs de haut niveau [27]. En ce qui concerne l'ovalisation de l'acetabulum, outre l'étude d'Erickson et al. [20], l'étude biomécanique du cavalier permet d'expliquer cette adaptation. En effet, la position théorique du cavalier, le " rein voussé » (rétroversion du bassin), oriente les acetabula vers l'avant et induit une mise en tension accrue des muscles fléchisseurs et adducteurs de la hanche $[59,60]$. Par conséquent, la position " rein voussé », source d'épargne pour le rachis, implique une poussée des têtes fémorales sur les acetabula.

L'analyse du sujet de la sépulture 11 met en avant l'intérêt de certains marqueurs (notamment l'ovalisation de l'acetabulum), et les données de la littérature médicales attestent de

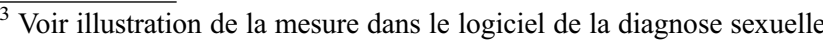
probabiliste (DSP) de Murail et al. [46].

${ }^{4}$ Données pour 29 hommes et 29 femmes aimablement fournies par J. Brůžek.
} 


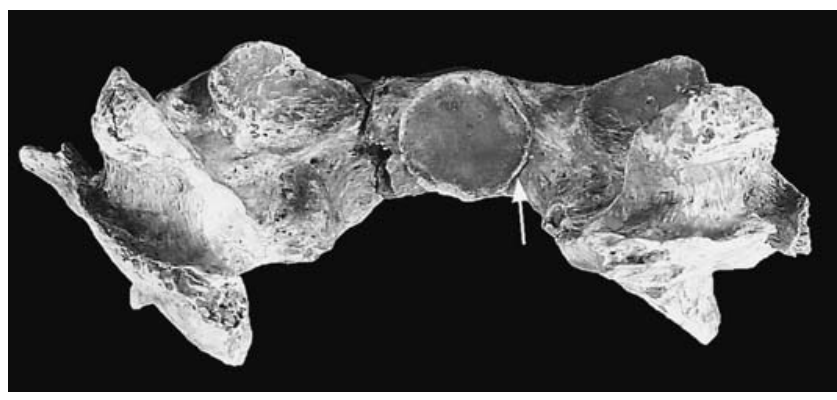

Fig. 4 Déformation mineure du contour de la fovea dentis de l'atlas / Minor deformation of the fovea dentis.

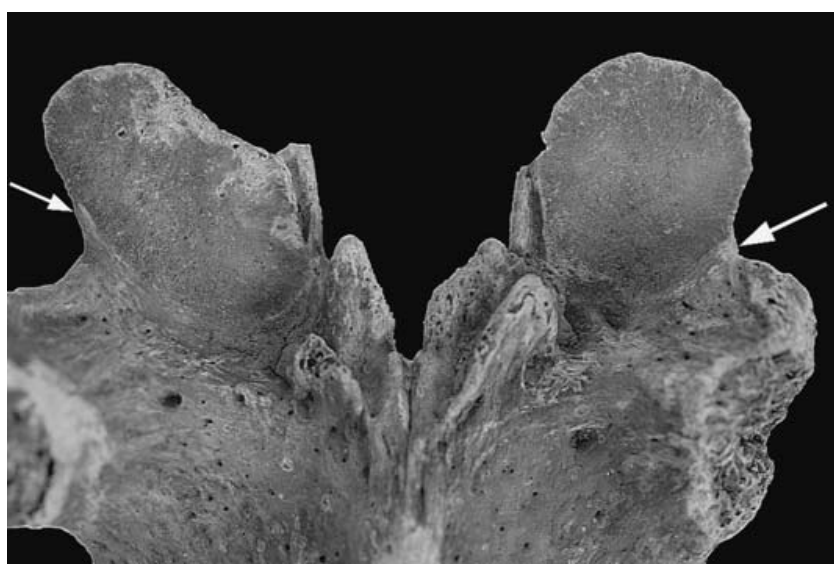

Fig. 5 Déformation mineure du contour des articulations postérieures d'une vertèbre thoracique / Minor deformation of the thoracic apophyseal joints

leur pertinence. Toutefois, l'étude de ce seul squelette, pour lequel la conservation osseuse est médiocre et les atteintes sont mineures, ne suffit pas à établir un ensemble lésionnel fiable de la pratique cavalière. Les études des MOA qui se fondent sur un référentiel actuel (transposition) imposent le postulat d'une biomécanique invariante: la gestuelle ancienne serait similaire à celle de nos jours [24]. Peu d'activités sont transposables de cette manière [6,61]. De plus, un marqueur isolé peut difficilement être caractéristique d'une activité donnée. Plusieurs activités peuvent produire une même lésion, et la même activité peut entraîner des atteintes différentes suivant les individus [5]. L'équitation est une pratique au sein de laquelle l'équipement du cavalier s'est modifié au cours du temps pour améliorer l'assise du cavalier au combat. La citation de l'auteur grec Xénophon [15] permet d'entrevoir les traumatismes causés par la monte suivant le type de siège, la position inconfortable imposée au cavalier et, plus généralement, l'influence du matériel équestre et de l'assise du cavalier sur le corps de ce dernier. Point sur lequel s'accordent les études médicales [62-64]. Pour notre sujet, le référentiel actuel est le cavalier professionnel

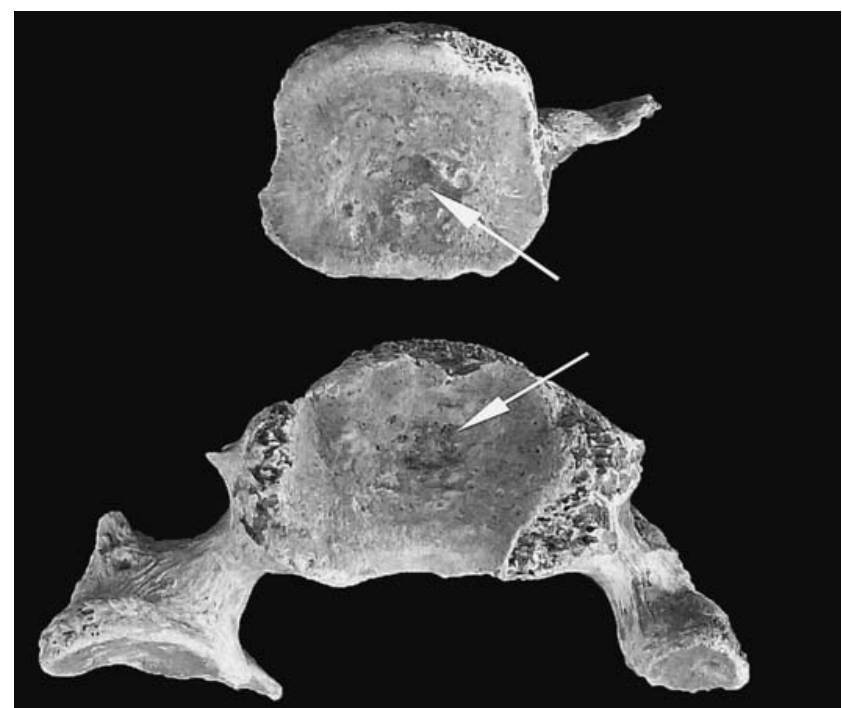

Fig. 6 Trace d'une ostéochondrose intervertébrale modérée sur les corps de deux vertèbres cervicales / Moderate intervertebral osteochondrosis on two cervical vertebrae
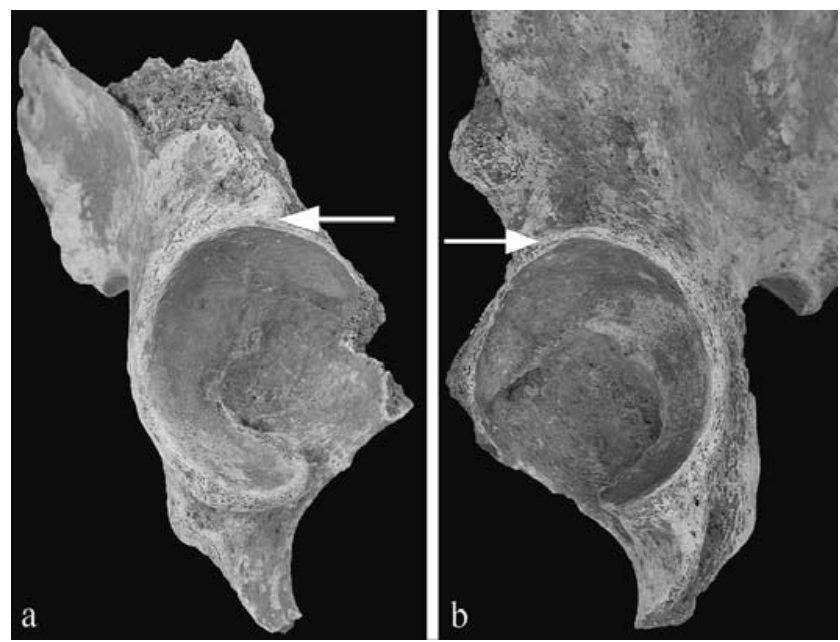

Fig. 7 Étirement vers le haut du bord antérosupérieur des acetabula droit (a) et gauche (b) / Upward elongation of the right(a) and left (b) acetabula

avec ses pathologies en rapport avec le type de matériel équestre utilisé par ce dernier.

Outre le fait d'attester l'adéquation de certains marqueurs osseux, observés sur le sujet de la sépulture 11, avec la pratique cavalière, les études médicales mettent, également, en avant d'autres indicateurs ostéologiques potentiels. L'excès de la pratique équestre chez le cavalier professionnel se traduit par des lésions périarticulaires et articulaires de la hanche et des atteintes du rachis [27]. Dans son traité d'anatomie humaine, Paturet définit les muscles adducteurs comme « les 


\begin{tabular}{|lll|}
\hline $\begin{array}{l}\text { Tableau } 3 \\
\text { droit et gauche / Vertical acetabular diameter }(\text { VEAC) and hori- }\end{array}$ \\
\begin{tabular}{l} 
zontal acetabular diameter $(H O A C)$ \\
\hline Acetabulum
\end{tabular} & VEAC (mm) & HOAC (mm) \\
\hline $\begin{array}{l}\text { Droit } \\
\text { Gauche }\end{array}$ & 60,44 & 54 (valeur estimée) \\
& 58,88 & 54,5 (valeur estimée) \\
\hline
\end{tabular}

Tableau 4 Longueur et largeur des fovéas droite et gauche / Length and width of the right and left fovea capitis

\begin{tabular}{|lll|}
\hline Fovéa & Longueur $(\mathbf{m m})$ & Largeur $(\mathbf{m m})$ \\
\hline Droite & 22,03 & 13,29 \\
Gauche & 22,56 & 13,00 \\
\hline
\end{tabular}
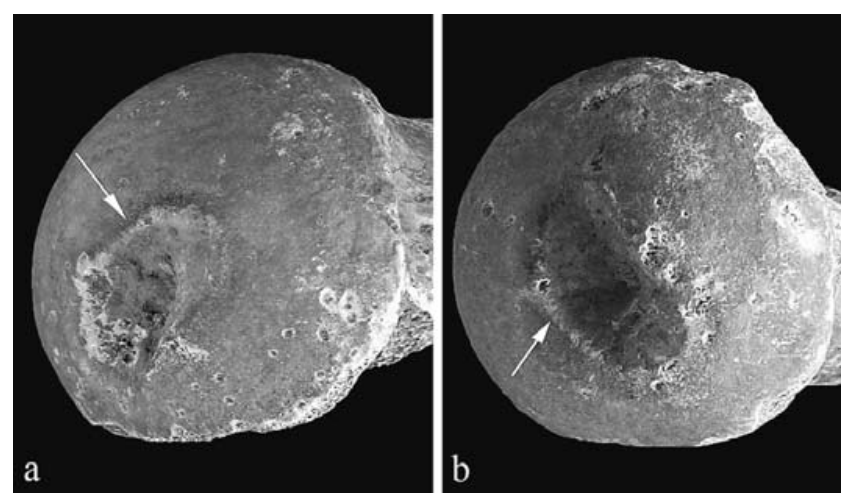

Fig. 9 Ostéophytoses périfovéales en bourrelet des fovéas droite (a) et gauche (b) / Marginal osteophytes of the right (a) and left (b) fovea capitis

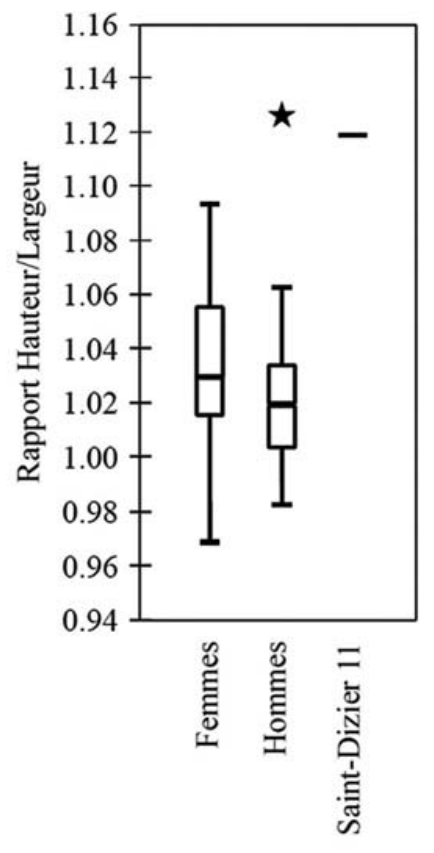

Acetabulum gauche

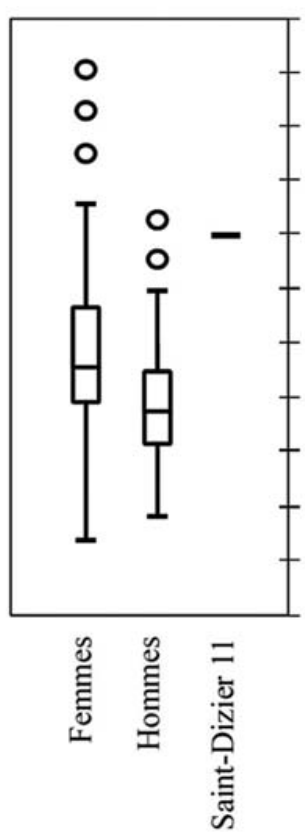

- Médiane

D $15 \%-175 \%$

I Etendue Non-Atypique

○ Atypiques

$\star$ Extrêmes

Fig. 8 Rapport hauteur/largeur des acetabula droit et gauche. Ce rapport est proche de 1 dans l'échantillon de référence / Ratio of vertical to horizontal acetabular diameter. The vertical and horizontal acetabular diameters are similar in the reference sample

muscles du cavalier » [65]. En équitation, la pratique de stages intensifs et les longues séances de mise en selle, notamment sans étrier, favorisent particulièrement les atteintes des tendons des adducteurs qui se traduisent radiographiquement par un aspect crénelé sur la branche ischiopubienne [27]. Ces enthésopathies concernent principalement la tranche d'âge 20-30 ans. L'atteinte peut être bilatérale ou unilatérale, et les muscles les plus souvent touchés sont le muscle gracile et le muscle long adducteur au niveau de l'insertion pubienne $[28,66]$. Sur le rachis lombaire du cavalier profes- sionnel, on observe une fréquence élevée d'hyperlordose lombaire de type renversement postérieur $(51 \%)[63,64]$. Cette pathologie est encore plus fréquente lorsque la pratique de l'équitation a commencé très jeune et de façon intense [57]. La DRC ${ }^{5}$ est la deuxième lésion du rachis fréquemment

\footnotetext{
$\overline{5 \text { Le siège de }}$ cette maladie est essentiellement thoracique. Elle provoque une cyphose (un dos rond) qui devient, si elle n'est pas traitée, irréductible une fois la croissance terminée. Elle affecte exceptionnellement la région supérieure lombaire [67].
} 
observée chez les cavaliers actuels [63]. Le cavalier subit de nombreux microtraumatismes répétés dans l'axe rachidien au cours des séances d'équitation. Ces contraintes mécaniques favorisent l'apparition de DRC et entraînent des déformations caractéristiques sur le rachis thoracique lorsque la pratique intensive de l'équitation est commencée avant la puberté $[59,67,68]$. Les DRC par leur prévalence $(50$ à $70 \%$ ), et notamment celle avant l'âge de neuf ans (70 \%), semblent être un bon indicateur pour identifier une population où cette pratique fait partie intégrante du mode de vie [63]. L'étude de Üstündag et Deveci illustre cette idée [23] : le rachis thoracique d'un jeune sujet féminin présente l'ensemble des déformations caractéristiques de cette atteinte, ainsi qu'une ovalisation de ses deux acetabula.

\section{Conclusion}

Les résultats de cette réflexion méthodologique confirment l'intérêt d'une démarche interdisciplinaire (archéologie, anthropologie biologique et données médicales) pour une meilleure reconnaissance des lésions liées à la pratique équestre. Les marqueurs osseux potentiels à retenir, mis en lumière par cette étude anthropologique et les données médicales, sont : les modifications osseuses relatives à l'arthrose rachidienne, les nodules de Schmorl, les DRC, l'hyperlordose lombaire, l'ovalisation de l'acetabulum, les ostéophytoses périfovéales et les enthésopathies sur le pubis. Parmi ces derniers, deux marqueurs sont particulièrement intéressants : les DRC et l'ovalisation de l'acetabulum. En effet, ils ne sont pas associés aux phénomènes dégénératifs (contrairement aux enthésopathies et à l'arthrose), et l'âge ne semble pouvoir être une cause de faux-positifs. Toutefois, ces modifications osseuses ne peuvent être désignées comme des critères suffisants pour la reconnaissance de la pratique équestre lorsque celles-ci sont observées de manière isolée. Il convient, également, d'entamer une réflexion à propos de l'influence de l'assise et du matériel équestre (en tenant compte de son évolution au court du temps) sur le corps du cavalier.

Remerciements Nous tenons à remercier, particulièrement, Armelle Alduc Le Bagousse, Robin Bendrey, Joël Blondiaux, Jaroslav Brůžek, Philippe Charlier, Patrice Courtaud, Sylvie Culot, Nicolas David, Paul-Henri Francfort, Véronique Gallien, Dominique Henri-Gambier, Hervé Guy, Michel Kazanski, Sylvie Kliesch-Pluton, Benoit Lagache, Elizabeth Miller, Pascal Murail, Stéphane Rottier, Bernadette Schnitzler, Alain Tourneux et Michaël Wiss pour leur concours à la réalisation de cette recherche. Nous remercions, également, les deux lecteurs anonymes pour leurs remarques et conseils, pertinents et avisés, dans la présentation de ce travail.

\section{Références}

1. Outram AK, Stear NA, Bendrey R, et al (2009) The Earliest Horse Harnessing and Milking. Science 323:1332-5

2. Brun P, Bridault A, Charpy JJ, et al (2001) Le cheval, symbole de pouvoirs dans l'Europe préhistorique. Musée de la préhistoire d'île-de-France, Nemours, France, 104 p

3. Pálfi G (1992) Traces des activités sur les squelettes des anciens hongrois. Bull Mem Soc Anthropol Paris 4(3-4):209-31

4. Reinhart KJ, TIezen L, Sandness KL, et al (1994) Trade, contact, and female health in Northeast Nebraska. In: Larsen CS, Milner GR (eds) In the wake of contact, biological responses to conquest. Wiley-Lyss Press, New York, pp 63-74

5. Dutour O (1992) Activités physiques et squelette humain : le difficile passage de l'actuel au fossile. Bull Mem Soc Anthropol Paris, 4(3-4):233-41

6. Villotte S (2008) Les marqueurs ostéoarticulaires d'activité. In: Charlier P (ed) Ostéoarchéologie et techniques médicolégales tendances et perspectives. Pour un «manuel pratique de paléopathologie humaine ». De Boccard, Paris, pp 383-9

7. Villotte S (2009) Enthésopathies et activités des hommes préhistoriques, recherches méthodologiques et application aux fossiles européens du paléolithique supérieur et du mésolithique. BAR International Series 1992, Oxford, Archaeopress, England, $206 \mathrm{p}$

8. Lebedynsky L (2007) Les nomades : les peuples nomades de la steppe des origines aux invasions mongoles : $\mathrm{I}^{\mathrm{e}}$ siècle av J.-C.$\mathrm{XIII}^{\mathrm{e}}$ ap J.-C. Errance, Paris, France, $301 \mathrm{p}$

9. Perin P (1970) Quelques objets exceptionnels provenant des tombes de chefs du cimetière mérovingien de Mézières. Rev Hist Ardennaise 4:71-7

10. Perin P (1972) Trois tombes de « chefs » du début de la période mérovingienne : les sépultures $\mathrm{n}^{\text {os }} 66,68$ et 74 de la nécropole de Mézières (Ardennes). Bull Soc Arch Champenoise 3-70

11. Lannois A (1896) Le cimetière gallo-franc de Thugny. Rev Hist Ardennaise 3:5-9

12. Meyer O, Coxall DJ, Heron C, et al (1985) Rapport de fouilles archéologiques 1983, ville de Saint-Denis. Saint-Denis, France

13. Lazaris S (2005) Considérations sur l'apparition de l'étrier : contribution à l'histoire du cheval dans l'Antiquité tardive. In: Gardeisen A (ed) Les équidés dans le monde méditerranéen antique. Acte du colloque organisé par l'École française d'Athènes, le centre Camille-Jullian et l'UMR 5140 du CNRS, Athènes, 26-28 novembre 2003. Édition de l'Association pour le développement de l'archéologie en Languedoc-Roussillon, Lattes, pp 275-88

14. Bona I (2002) Les Huns, le grand empire barbare d'Europe IV ${ }^{\mathrm{e}}$ $\mathrm{v}^{\mathrm{e}}$ siècles. Errance, Paris, France, $239 \mathrm{p}$

15. Xenophon (1978) De l'art équestre, texte établi et traduit par E. Delebecque. Les Belles lettres, Paris, France

16. Pálfi G (1997) Maladies dans l'Antiquité et au Moyen Âge. Paléopathologie comparée des anciens Gallo-Romains et Hongrois. Bull Mem Soc Anthropol Paris, 9(1-2):1-206

17. Alduc-le-Bagousse A, Blondiaux J, Pilet C (1992) La dame d'Hochfelden. Cahiers alsaciens d'Arch, d'Art et d'Hist, tome XXV, 75-90

18. Blondiaux J (1994) À propos de la dame d'Hochfelden et de la pratique de la cavalière : discussion autour des sites fonctionnels fémoraux. In: Buchet L (ed) La femme pendant le Moyen Âge et l'époque moderne. Actes des $6^{\mathrm{e}}$ journées anthropologiques (9-11 juin 1992), dossier de documentation archéologique $n^{\circ} 17$, Valbonne, pp 97-109

19. Courtaud P, Rajev D (1998) Osteomorphical features of nomadic riders: some examples from Iron Age populations located in Southwestern Siberia. In: Pearce M, Tosi M (eds) Papers from 
the EAA Third annual meeting. Oxford, BAR International Series 717, Ravenna, pp 110-3

20. Erickson JD, Lee DV, Bertram JEA (2000) Fourier analysis of acetabulum shape in Native American Arikara Populations before and after acquisition of horses. Am J Phys Anthropol 113:473-80

21. Charlier P (2002) Ostéoarchéologie humaine de deux nécropoles étrusco-celtiques (Monte Bibele et Monterenzio Vecchio, Italie). École pratique des hautes études, IV ${ }^{\mathrm{e}}$ section, Sciences historiques et philologiques, pp 173-77

22. Fornaciari G, Giusiani S, Vitiello A (2003) Paleopatologia del cimitero signorile del castello di Monte di Croce (I a fase, XI secolo). III Congresso Nazionale di Archeologia Medievale, Edizioni All'Insegna del Giglio, Firenze, pp 292-8

23. Üstündag H, Deveci A (2011) A possible case of Scheuermann's disease from Akarcay Höyük, Birecik ( $\square$ anliurfa, Turkey). Int J Osteoarchaeol 21:187-96

24. Crubezy E, Causse L, Delmas J, et al (1998) Le paysan médiéval en Rouergue. Cimetière et église de Canac (Campagnac, Aveyron). Musée archéologique de Montrozier, Montrozier, France, $263 \mathrm{p}$

25. Pálfi G, Dutour O (1995) Informations sur les activités du passé apportées par le squelette. Dossiers d'archéologie 208:12-21

26. Villotte $S$ (2006) Connaissances médicales actuelles, cotation des enthésopathies : nouvelles méthodes. Bull Mem Soc Anthropol Paris, ns, t 18, fasc $1-2,65-85$

27. Auvinet B (1980) La hanche du cavalier. Med Sport 54/5:281-5

28. Janssen B (2008) Lettre d'information : adducteur.com

29. Truc MC, Achard-Corompt N, Ahü-Delor A, et al (2009) SaintDizier (Haute-Marne) «La Tuilerie - RN4 - Aire de service ", sépultures aristocratiques et habitat du haut Moyen Âge. Rapport de fouille archéologique préventive, Inrap, Champagne-Ardenne, France, 4 volumes

30. Lepage L (1970) Fouille d'un habitat gallo-romain à Saint-Dizier « Les Crassées ». Mem Soc des Lettres, des Sciences, des Arts, de l'Agriculture et de l'Industrie de Saint-Dizier, t II, 3-82

31. Durost R, Achard-Corompt N, Truc MC (2004) Saint-Dizier (Haute-Marne), route de Joinville "Les Crassées », parcelle DL 153, Opération archéologique préventive, rapport de diagnostic. Châlons-en-Champagne, DRAC de Champagne-Ardenne, France, $35 \mathrm{p}$

32. Beague-Tahon N, Bourdin D (1993a) Rapport de fouilles archéologiques réalisées au carrefour giratoire de la Marina, RN67, entrée Sud-Est de Saint-Dizier (Haute-Marne). Service régional de l'archéologie de Champagne-Ardenne, France

33. Beague-Tahon N, Bourdin D (1993b) Rapport de fouilles archéologiques réalisées au centre d'activités commerciales et tertiaires du Chêne Saint-Amand, lieu-dit « Le Seugnon-Toupot-Millot » à Saint-Dizier (Haute-Marne). Service régional de l'archéologie de Champagne-Ardenne, France

34. Böhner K (1959) Das Grab eines fränkischen Herren aus Morken im Rheinland. Führer des rheinischen Landesmuseums in Bonn, $\mathrm{n}^{\mathrm{o}} 4,46 \mathrm{p}$

35. Stein F (1993) Grabkammern bei Franken und Alamanen, Beobachtungen zur sozialen Gliederung und zu den Verhältnis nach der Eingliederung der Alamanen in das merowingische Reich. Herrschaft, Kirche und Kultur, Beiträge zur Geschichte des Mittelalters, Festschrift für Friedrich Prinz zur seinem 65, Geburtstag, Sonderdruck, $41 \mathrm{p}$

36. Yvinec JH (2009) La sépulture de cheval, étude biologique. In: Truc MC (ed) Saint-Dizier « La Tuilerie - RN4 - Aire de service ", sépultures aristocratiques et habitat du haut Moyen Âge. Rapport de fouille archéologique préventive, Inrap, volume 1, Champagne-Ardenne, pp 134-5

37. Tacite (1949) La Germanie, texte établi et traduit par J. Perret. CUF, Série Latine, Paris
38. Salin E (1959) La civilisation mérovingienne, d'après les sépultures, les textes et le laboratoire, quatrième partie: les croyances, conclusions, index général. A et J Picard, Paris, France, 579 p

39. Koch U (1996) Stätten der Totenruhe-Grabformen und Bestattungsitten der Franken. In: Zabern (von) P (ed) Die Franken, Wegbereiter Europas: vor 1500 Jahren: König Chlodwig und seine Erben. Catalogue de l'exposition itinérante, pp 723-38

40. Dierkens A, Le Bec C, Perin P (2008) Sacrifice animal et offrandes alimentaires en Gaule mérovingienne. In: Lepetz S, Van Andringa $\mathrm{W}$ (eds) Archéologie du sacrifice animal en gaule romaine. Rituels et pratiques alimentaires, Archéologie des plantes et des animaux, 2. Éditions Monique Mergoil, Montagnac, pp 279-99

41. Perin P (1997) L'expansion franque (vers 486-vers 540). In: Perin P, Kazanski M (eds) Les Francs, précurseurs de l'Europe. Musée du Petit Palais, Paris, pp 70-83

42. Evison V (1967) The Dover Rings-sword and Other Sword-rings and beads. Communicated to the Society of Antiquaries, tome CI, 63-118

43. Perin P (2008) Les tombes de « chefs " mérovingiennes. Archaeologia 461:40-2

44. Leroy M, Merluzzo P (1998) Les ateliers sidérurgiques médiévaux de Saint-Dizier (Haute-Marne). Bull Soc Arch Champenoise, France, tome 91, $\mathrm{n}^{\mathrm{o}} 4,126 \mathrm{p}$

45. Brůžek J (2002) A method for visual determination of sex, using human hip bone. Am J Phys Anthropol 117:157-68

46. Murail P, Brůžek J, Houët F, et al (2005) DSP: a tool for probabilistic sex diagnosis using worldwide variability in hip bone measurements. Bull Mem Soc Anthropol Paris, 17(3-4):167-76

47. Schmitt A (2005) Une nouvelle méthode pour estimer l'âge au décès des adultes à partir de la surface sacropelvienne iliaque. Bull Mem Soc Anthropol Paris, 17(1-2):89-101

48. Masset C (1982) Estimation de l'âge au décès par les sutures crâniennes. Thèse en sciences naturelles, université de Paris-VII, Paris, France $298 \mathrm{p}$

49. Moorrees CFA, Fanning EA, Hunt EE (1963) Age variation of formation stages for ten permanent teeth. J Dent Res 42, 6:1490-502

50. Birkner R (1980) L'image radiologique typique du squelette. Aspect normal et variantes chez l'adulte et l'enfant, Maloine, Paris, France

51. Trotter M, Gleser G (1958) A re-evaluation of estimation of stature based on measurements of stature takenduring life and long bones after death. Am J Phys Anthropol 16:79-124

52. Paresys C (2010) Savigny-sur-Ardres « la Croix Cassée ». Rapport final d'opération, Inrap, SRA Châlons-en-Champagne, DRAC Champagne-Ardenne, France

53. Rogers J, Waldron T (2001) DISH and the monastic way of life. Int J Osteoarchaeol 11, 5:357-65

54. Murail P (2005a) Variations anatomiques non métriques : les caractères discrets. In: Dutour O, Hublin J, Vandermeersch B (eds) Objets et méthodes en paléoanthropologie (Orientations et méthodes, 7). Comité des travaux historiques et scientifiques, Paris, pp $137-51$

55. Bräuer G (1988) Osteometrie. In: Knussmann R (ed) Anthropologie, Handbuch der vergleichenden Biologie des Menschen. Band I, Gustav Fisher Verlag, Stuttgart, pp 160-232

56. Komar DA, Grivas C (2008) Manufactured population: what do contemporary reference skeletal collections represent? A comparative study using the Maxwell Museum Documented Collection. Am J Phys Anthropol 137:224-33

57. Lang N (1995) Pathologie du rachis chez le cavalier. Thèse de médecine, université de Créteil, Créteil, France, $70 \mathrm{p}$

58. Demarais Y, Lequesne M (1979) La hanche du sportif. Gaz Med France 86:2969-79

59. Auvinet B, Estrade M (1998) La santé du cavalier, conseils pratiques pour une équitation sans risque. Chiron, Paris, France, $144 \mathrm{p}$ 
60. Maistre B (1995) Cheval et sport. Med Sport, hors série, tome 69, 143-6

61. Dutour O (2000) Chasse et activités physiques dans la préhistoire : les marqueurs osseux d'activités chez l'homme fossile. Anthropologie et Préhistoire, 111, pp 156-65

62. Quinn S, Bird S (1996) Influence of saddle type upon the incidence of lower back pain in equestrian riders. Brit J Sport Med, 30, pp 140-4

63. Auvinet B (1999) Lombalgies et équitation. Synoviale, rhumatologie sportive, $\mathrm{n}^{\mathrm{o}} 83,25-31$
64. Pognant C (2006) Lombalgie chez les cavaliers, étude épidémiologique et revue de la littérature. Thèse de médecine, université Claude-Bernard de Lyon, Lyon, France, $70 \mathrm{p}$

65. Paturet G (1951) Traité d'anatomie humaine, tome II : Membres supérieurs et inférieurs. Masson, Paris, France, $1115 \mathrm{p}$

66. Auvinet B (1997) Pathologies pubo-inguinales du cavalier. Med Sport, Expansion scientifique française, 1-2

67. Goussard J (1998) La maladie de Scheuermann. Sport et Médecine Actualité, 1-10

68. Diard F, Chateil JF, Brun M (2000) La maladie de Scheuermann. www.dfip-radiopediatrie.org 